\title{
Comparison of Synthesis Strategies for a Dual-Polarized Reflectarray
}

\author{
Loic Marnat, ${ }^{1}$ Renaud Loison, ${ }^{1}$ Raphaël Gillard, ${ }^{1}$ Daniele Bresciani, ${ }^{2}$ and Hervé Legay ${ }^{2}$ \\ ${ }^{1}$ Antennes et Hyperfréquences, Institut d'Electronique et de Télécommunications de Rennes, UMR CNRS 6164, \\ INSA, 20 Avenue des Buttes de Coësmes, CS 70839, 35708 Rennes, France \\ ${ }^{2}$ Thales Alenia Space Toulouse, 26 Avenue JF Champollion, BP 1187, 31037 Toulouse Cedex, France
}

Correspondence should be addressed to Loic Marnat, loic.marnat@kaust.edu.sa

Received 30 January 2012; Accepted 14 May 2012

Academic Editor: Manuel Arrebola

Copyright () 2012 Loic Marnat et al. This is an open access article distributed under the Creative Commons Attribution License, which permits unrestricted use, distribution, and reproduction in any medium, provided the original work is properly cited.

\begin{abstract}
This paper presents and compares four optimization strategies for printed reflectarrays. They are applied to synthesize a dualpolarized reflectarray using two kinds of slot-loaded patches. They provide objective criteria to deal with the numerous geometrical parameters available to tune the phase. The study relies on the comparative measurements of four different prototypes for the same $22 \lambda_{0} \times 22 \lambda_{0}$ reflectarray (with $\lambda_{0}$ the wavelength at $f_{0}=14.25 \mathrm{GHz}$ ).
\end{abstract}

\section{Introduction}

A reflectarray antenna combines advantages of reflectors and phased array antennas. Indeed, neither lossy feeding network nor heavy and cumbersome profile are involved. A reflectarray antenna is composed of a primary feed lighting elementary cells that delay the impinging wave and permit to shape the radiated field. In most cases, the reflected phase is controlled by the geometry or orientation of the radiating elements $[1,2]$, stubs or delay lines [3], or the use of reconfigurable material [4]. To achieve the phase law all over the reflectarray surface, the unit cell must be able to cover the whole $360^{\circ}$ phase range. In [3], a reflectarray has been synthesized with a multiple layer structure allowing a true time delay compensation. A large bandwidth has then been achieved $20(\%)$. Single-layer reflectarrays are usually more limited in term of bandwidth. The required $360^{\circ}$ phase range is more difficult to obtain without a strong resonance which introduces more losses. On the other hand, single substrate layer is cost effective and easy to manufacture.

In this paper, the used radiating element is a patch loaded with slots (Figure 1), having dual polarization capabilities [5]. It is an extension of the single-polarized patch structure introduced in $[6,7]$. Furthermore, a reconfigurable cell can be obtained by controlling slots with PIN diodes or MEMS
$[8,9]$. The considered cell design is defined with six parameters for each polarization. As a consequence, a large number of cell designs are available to produce a given phase shift. This amount of solutions is advantageous for an accurate reflectarray synthesis and it also increases its complexity.

The aim of this paper is to present and validate an original reflectarray layout synthesis. This synthesis technique is based on specific cell selection strategies. Three strategies are proposed and compared. In the first part of the paper, the used elementary cell is described and its RF characteristics are studied. The proposed synthesis process is presented in the second part. In the third one, it is applied to a specific space telecommunication antenna configuration. Finally, the measurement of the resulting reflectarray are presented.

\section{Elementary Cell}

2.1. Description. Figure 1 presents the proposed dualpolarized cells. Two cell configurations are considered. Figures 1(a) and 1(b) depicts the centered slots configuration (defined by a microstrip square patch loaded with a Jerusalem cross slot) and the emerging slots configuration (defined by two pairs of orthogonal slots located at the edges of the microstrip square patch), respectively. For each 

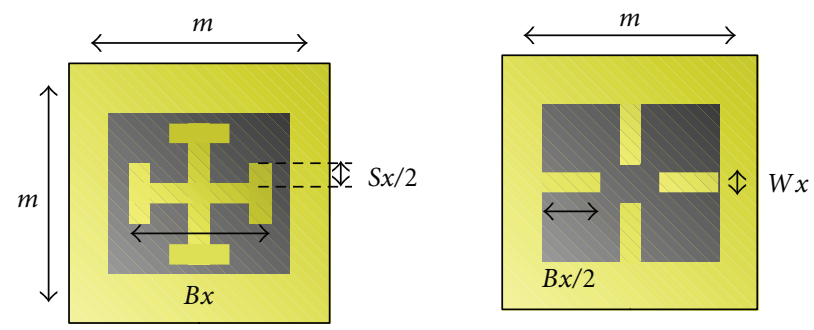

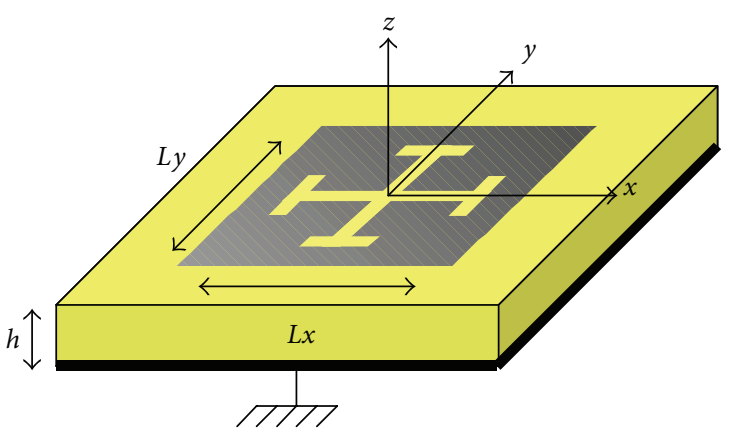

(a)

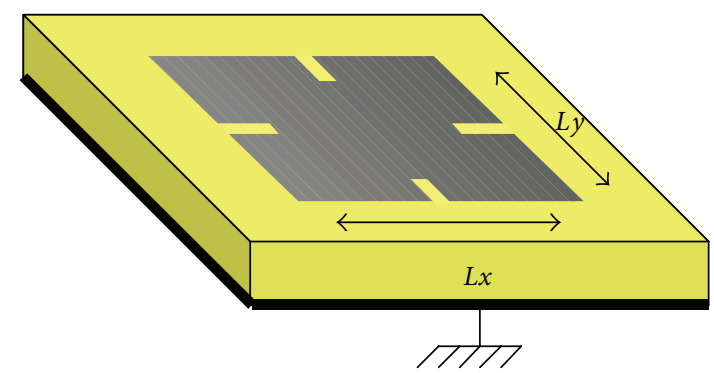

(b)

Figure 1: Patch loaded by (a) centered crossed slots or (b) emerging slots ( $\left.h=4 \mathrm{~mm}, \varepsilon_{r} \simeq 1\right)$.

polarization, the phase reflected by the cell is tuned by varying six geometrical parameters characterizing the cell design in each direction: the patch size ( $L x$ and $L y$ ), the slot length ( $B x$ and $B y)$ and width ( $W x$ and $W y$ ), the stub length ( $S x$ and $S y$ ) and width (WSx and $W S y$ ), and the slot configuration (centered crossed and emerging).

2.2. Analysis of the Reflected Phase. The phase response of the cell is simulated for a linearly polarized incident plane wave under normal incidence with a self-developed 2.5D electromagnetic simulator. It has been specifically developed for planar self-similar structures and validated through different simulation comparisons and measurements [10]. Floquet hypotheses are used in order to take mutual coupling effects into account while keeping a reasonable simulation time. In these conditions, the cell is assumed to be extracted from an infinite periodic array. A $y$ polarized incident field is applied and the phase computed is the one of the direct reflection coefficient: $\angle \Gamma_{y y}$. In the following, this achieved phase is noted $\phi^{\text {ach }}$.

Figure 2 presents the phase shift for the centered slots configuration (Figure 1(a)) versus the slots lengths for different patch dimensions. In this section, a symmetrical cell is considered which means the $x$ and $y$ cell parameters are equal. The size of the array lattice is set to $0.5 \lambda_{0}$ at $f_{0}=$ $14.25 \mathrm{GHz}(m=10.526 \mathrm{~mm})$. The substrate is a $4 \mathrm{~mm}$ thick composite panel whose equivalent dielectric constant is approximated by 1 .

Two areas can be identified in Figure 2. The first one corresponds to short slots ( $B x$ smaller than $4 \mathrm{~mm}$ ) with a phase shift which is mainly controlled by the size of the patch. In the second one, where $B x$ is greater than $4 \mathrm{~mm}$, the slot
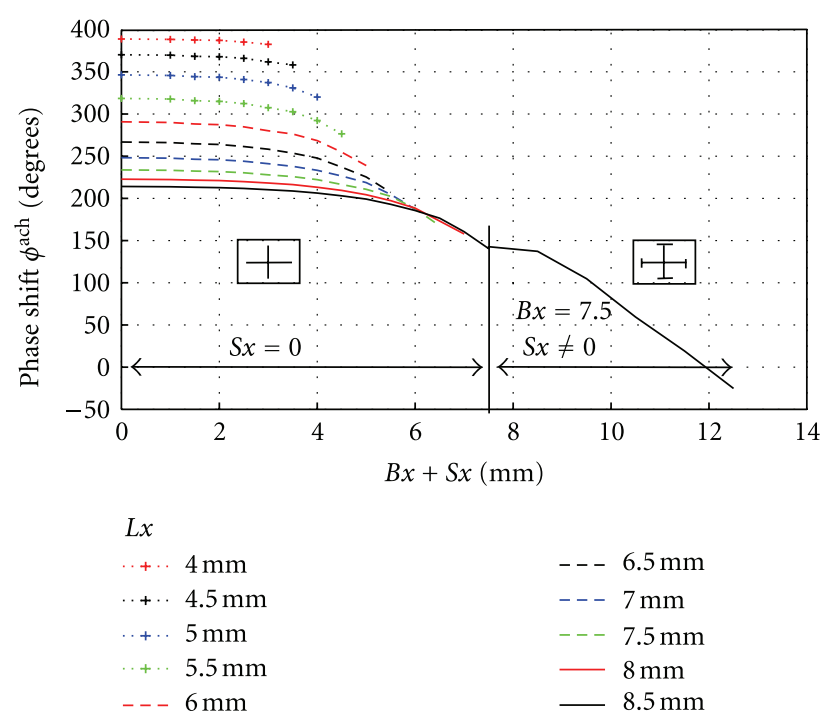

FIGURE 2: Phase shift versus slots lengths for different patch sizes $(L x)$ at $f_{0}=14.25 \mathrm{GHz}$ in normal incidence, and $W x=0.9 \mathrm{~mm}$.

length becomes the most effective tuning parameter. The addition of stubs (here, only for $L x=8.5 \mathrm{~mm}$ and $B x=$ $7.5 \mathrm{~mm}$ ) permits to extend the phase range up to $414.1^{\circ}$. Note that the phase range could be increased further by using thinner slots (up to $548.5^{\circ}$ for $0.1 \mathrm{~mm}$ slot widths).

The same results are presented in Figure 3 for the emerging slots configuration (Figure 1(b)). It appears that the slot lengths are more influent for this cell configuration. Indeed, they directly affect the currents which are mainly 

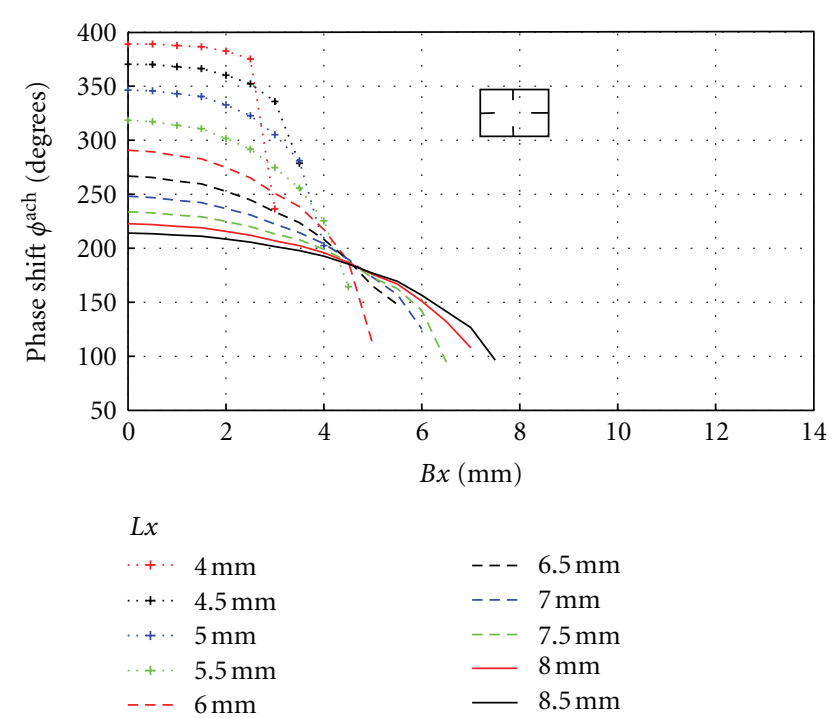

FIGURe 3: Phase shift versus slot lengths for different patch sizes $(L x)$ at $f_{0}=14.25 \mathrm{GHz}$ under normal incidence, and $W x=0.9 \mathrm{~mm}$.

concentrated on patch edges. These different behaviors motivate the use of the two complementary configurations. As will be seen later on, this provides an additional flexibility to the optimization process.

It can be highlighted that the considered cells are patches on air substrate which gives low loss elements. Nevertheless, the losses of the centered crossed slots configuration are higher when stubs are used due to the resonance of the cell which is shifted close to the operating frequency.

2.3. Dual-Polarization Capabilities. In this section, the dual polarization capabilities of the cell are investigated. As previously, the cell is excited by a $y$ polarized electric field. The phase of the reflected wave is thus controlled by the $x$ oriented main slot $(B x)$ and $y$ oriented stubs $(S x)$ referred to as the excited slots. The orthogonal slots defined by $B y$ and Sy should not affect the reflected wave and are referred to as the parasitic slots. The evolution of the phase of the direct reflection coefficient is plotted in Figure 4 as a function of the lengths of the excited slots at the central frequency. Different lengths of parasitic slots are considered for the two cell configurations.

This figure shows that the reflected phase can be tuned by varying the excited slots while the effect of the parasitic slots is negligible. As a consequence, both orthogonal linear polarizations can be controlled independently.

2.4. Cell Diversity. One key issue in the reflectarray layout synthesis is to select the appropriate design for each cell in order to comply with the desired antenna performance. One cell design is defined by six geometrical parameters for each polarization. Consequently, a large number of cell designs can be obtained, with several ones that produce the same phase shift. This diversity gives flexibility to the synthesis process but increases its complexity.

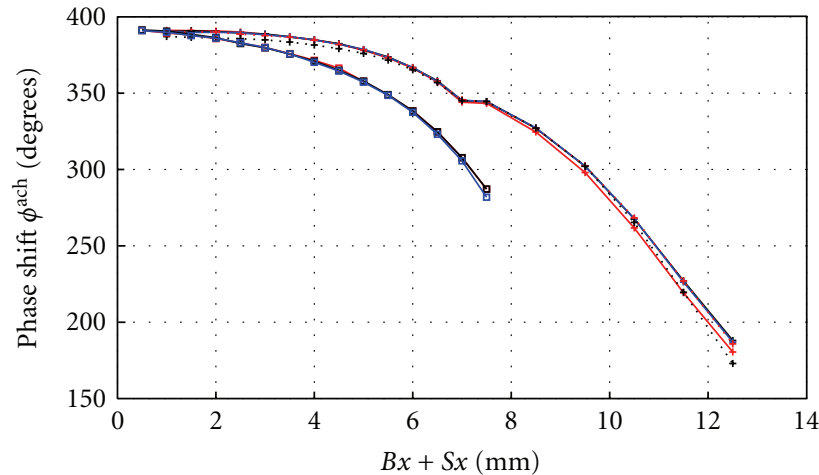

$$
\begin{aligned}
& B y+S y \\
& \longrightarrow 1.5 \mathrm{~mm}+0 \mathrm{~mm} \\
& -4 \mathrm{~mm}+0 \mathrm{~mm} \\
& \longrightarrow 6.5 \mathrm{~mm}+0 \mathrm{~mm} \\
& \text {.. } 6.5 \mathrm{~mm}+0.5 \mathrm{~mm} \\
& \begin{array}{ll}
\cdots+\cdots & 6.5 \mathrm{~mm}+1.25 \mathrm{~mm} \\
\rightarrow- & 0.75 \mathrm{~mm}+0 \mathrm{~mm} \\
\rightarrow & 2.25 \mathrm{~mm}+0 \mathrm{~mm} \\
\rightarrow & 3.75 \mathrm{~mm}+0 \mathrm{~mm}
\end{array}
\end{aligned}
$$

FIGURE 4: Effect of the parasitic slots $(B y+S y)$ on the phase of the direct reflection coefficient for the centered $(+)$ and the emerging ( $\square$ ) slots configurations at $f_{0}=14.25 \mathrm{GHz}$ under normal incidence, and $W x=0.9 \mathrm{~mm}$.

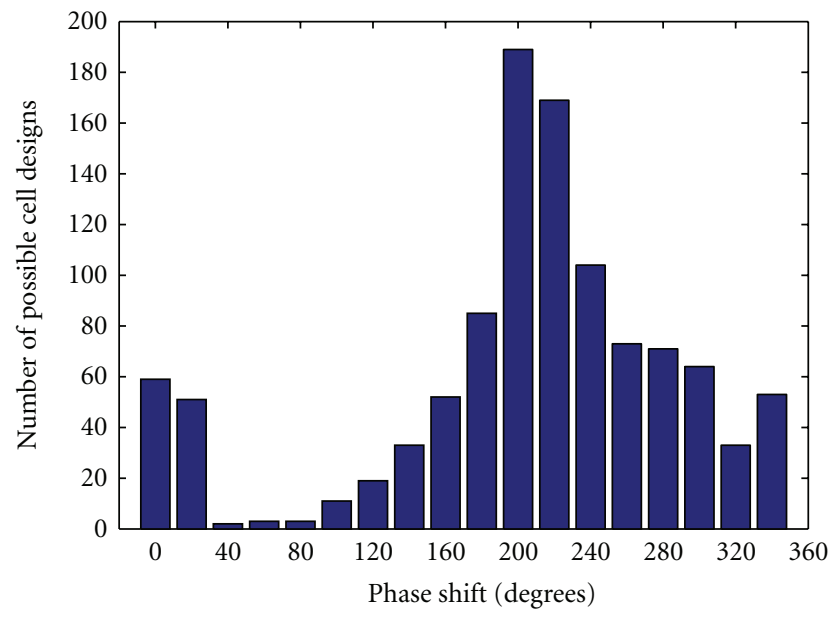

FIGURE 5: Number of cell designs producing a given range of phase shift $\left(20^{\circ}\right.$ by $\left.20^{\circ}\right)$ under normal incidence at $14.25 \mathrm{GHz}$ for a uniform sampling of the geometrical parameter.

As an illustration, we consider the situation where each geometrical parameter has been sampled so that 1074 different cell designs are obtained. The resulting phase distribution, corresponding to the number of cell designs producing a given phase shift under normal incidence, is plotted in Figure 5.

This figure shows the multiplicity of cell designs available to reach a given phase shift. However, it can be noted that this distribution is not uniform. For instance, a "pinched zone" can be observed from 40 to $100^{\circ}$ where only few cell designs are available. This restricts the possible choices in this phase range for the optimization process. Nevertheless, the phase distribution is different for each incident angle. At the end, for a given reflectarray, many layouts can be generated satisfying the phase law at the central frequency. For instance, 
in [5], up to $6.45 \times 10^{3187}$ distinctive layouts could theoretically be generated for the studied antenna.

In these conditions, cell selection strategies must be implemented in order to optimize the reflectarray layout.

\section{Synthesis Process}

3.1. Cell Selection Strategies. A cell selection strategy aims at choosing the best design for each element in the array. The minimum requirement is that the achieved phase at the central frequency, $f_{0}$, matches the desired one:

$$
\phi_{i}^{\text {ach }}\left(f_{0}\right)=\phi_{i}^{\text {des }}\left(f_{0}\right)
$$

for $i=1$ to $N$, where $N$ is the number of elements in the array.

The reference cell, defined as the starting point when filling up the layout, is arbitrarily located at the center of the array. The cells are then successively designed from the reference one and moving to the edges of the array. In this section, four cell selection strategies are proposed and described.

(i) The Random strategy only operates a random selection among available cell designs achieving the desired phase shift at $f_{0}$ without any consideration on the geometry or other electrical characteristics. In this paper, these layouts are called " $R$ layout" for "Random" strategy.

(ii) The Dispersion optimization consists in selecting cell designs that not only produce the required phase shifts at central frequency $\left(f_{0}\right)$ but also the best achievable ones at the bandwidth extremities $\left(f_{\min }\right.$ and $\left.f_{\max }\right)$. It is the most conventional strategy when designing large-bandwidth reflectarrays [11].

The best cell design, for the $i$ th array element, is the one that minimizes the phase error between the desired and the achieved ones:

$$
\varepsilon_{i}(f)=\left|\phi_{i}^{\mathrm{des}}(f)-\phi_{i}^{\mathrm{ach}}(f)\right|
$$

at $f=f_{\min }$ and $f_{\max }$.

In this paper, such layouts are called " $D$ layout" for "Dispersion" strategy.

(iii) The third strategy is called Geometrical optimization and has been introduced in [12]. The aim of this original strategy is to maximize the geometrical similarity between contiguous cells in the reflectarray layout. The goal is to comply with the used Floquet simulation hypotheses and thus improve the prediction accuracy of the antenna performance.

As it has been shown in Section 2, each geometrical parameter does not equally affect the phase shift. Thus, to quantify the similarity between two cells $i$ and $j$, a similarity factor is defined in which a different weight is attributed to each geometrical parameter:

$$
\mathrm{SF}_{(i, j)}=\sqrt{\alpha_{L x} \cdot\left(L x_{i}-L x_{j}\right)^{2}+\alpha_{L y} \cdot\left(L y_{i}-L y_{j}\right)^{2}+\cdots} .
$$

In this paper, $\alpha_{L x}=7$ (patch size), $\alpha_{B x}=4$ (slot length), $\alpha_{S x}=1$ (stub length), $\alpha_{W x}=10$ (slot width), and $\alpha_{W S x}=10$ (stub width). In other words, a variation of slot width $(W x)$ leads to a larger $\mathrm{SF}_{(i, j)}$ value than the same variation of stub length $(S x)$. The similarity between cell $i$ and its neighbors is noted $\overline{\mathrm{SF}}_{i}$. It is defined by averaging the similarity factor between this cell and each of its nearest neighbors (eight for a rectangular lattice). An iterative process is used that results in a layout which minimizes the $\overline{\mathrm{SF}}_{i}$ factor for each array element and thus ensures smooth geometrical variations. The first iteration initializes the geometry of each cell by minimizing the $\overline{\mathrm{SF}}_{i}$ factor only considering those of its 8 nearest neighbors that have already been defined. As a consequence, the geometry of the first cell is chosen arbitrarily (only complying with the desired phase). In the next steps, the cell's geometry is progressively updated until the average value of $\overline{\mathrm{SF}}_{i}$ converges to its lowest value.

In this paper, these layouts are called " $G$ layout" for "Geometrical" strategy.

(iv) The last proposed strategy is called MRC optimization for "Maximization of Reflection Coefficients" strategy. It has been developed to improve both the reflectarray losses and the cross-polarization level. To do so, the direct reflection coefficient magnitude, $\left|\Gamma_{y y}\left(f_{0}\right)\right|$, is maximized for each array element at the central frequency $\left(f_{0}\right)$.

The simple Random cell selection will be used to assess the relevance of these strategies for reflectarray synthesis.

3.2. Layout Selection. In addition to the proposed cell selection strategies, it is possible to synthesize layouts with different initial conditions associated to the reference cell. Indeed, any phase offset can theoretically be applied to the phase law in the radiating aperture without changing the associated radiation pattern. The phase of the reference cell at the central frequency, $\phi_{0}=\phi_{0}^{\text {des }}\left(f_{0}\right)$, determines the absolute phase values for all the array elements at this frequency. Thus, the phase of the reference cell can be chosen arbitrarily. In practice, however, its choice is not neutral [13]. As seen in Figure 5, the phase distribution is not uniform. Thus, a judicious $\phi_{0}$ value is one that maximizes the number of possible cell designs for most of the array elements.

Although this general principle is quite simple, its direct application is not straightforward as the phase distribution depends on the incident angle (in Figure 5, it is only given for the normal incidence). As a consequence, in the following, the optimization process is repeated for different $\phi_{0}$ values. Then, the solution with the best simulated performance is selected.

To do so, some indicators are defined as follows.

(i) The phase errors between the simulated and the desired phase shifts over the array at a given frequency $f$ are quantified by the following mean and standard deviation:

$\bar{\varepsilon}(f)=(1 / N) \sum_{i=1}^{N} \varepsilon_{i}(f)$ quantifies the mean phase errors over the array. Note that $\bar{\varepsilon}\left(f_{0}\right)=0$ as all proposed strategies match the specification at $f_{0}(1)$. 


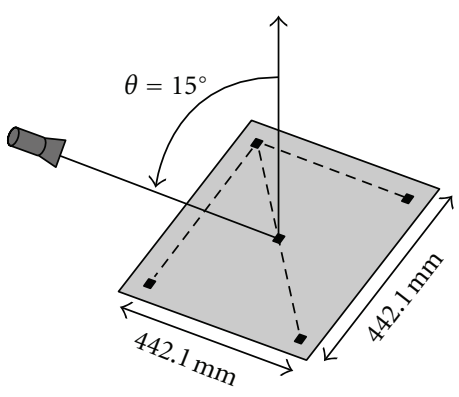

(a) Offset configuration

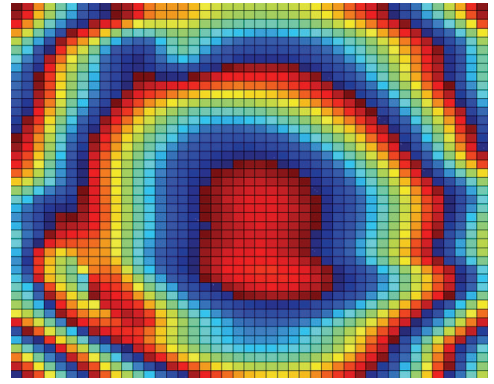

(b) Desired aperture phase shift at $f_{0}=$ $14.25 \mathrm{GHz}$

Figure 6: Antenna configuration $\left(F / D=1.3\right.$, maximum cell incidence angle $=38^{\circ}$, radiating aperture $=22 \lambda_{0} \times 22 \lambda_{0}$, and interelement spacing $\left.=0.5 \lambda_{0}\right)$.

(ii) The magnitude of the reflection coefficients is averaged over the array to quantify the ohmic losses and/ or the polarization conversions of the generated layout but does not account for the tapering of the illumination on the array. It is noted $\overline{\Gamma_{y y}}(f)$.

(iii) To quantify the similarity over the layout, a global similarity criterion, noted $\overline{\mathrm{SF}}$, is defined by averaging the $\overline{\mathrm{SF}}_{i}$ values all over the array.

Layouts can objectively be compared using these indicators and the best candidate can then be chosen.

\section{Application to a Specific Antenna Configuration}

4.1. RF Requirements. The synthesis process is applied to the design of a dual-polarized reflectarray antenna for spatial communications in the Ku band (from 14 to $14.5 \mathrm{GHz}$ ). The considered antenna configuration is depicted in Figure 6(a). The size of the antenna aperture is $22 \lambda_{0} \times 22 \lambda_{0}$. The $F / D$ ratio is set to 1.3 as a trade-off between frequency dispersion and spillover effects. Moreover, an offset configuration is chosen to limit feed blockage.

The same radiation pattern is required for both polarizations with a CONUS type coverage (for CONtinental United States). To get the phase shift required in the reflectarray aperture, a complex ratio between the field coming from the primary feed and the field reconstructed from the desired shaped radiation pattern in the aperture is performed. The desired aperture phase shift at $f_{0}=14.25 \mathrm{GHz}$ is shown in Figure 6(b).

\subsection{Layout Synthesis and Optimization}

4.2.1. Layouts Generation and Selection. Different layouts are generated using the four cell selection strategies. For each strategy, eight layouts are synthesized by considering eight initial conditions $\left(\phi_{0}\right)$ regularly distributed over the $360^{\circ}$ range. Among the eight layouts, the best one is then selected using the indicators defined in Section 3.2.
TABLE 1: Indicators of layouts synthesized with the $M R C$ strategy for eight $\phi_{0}$ values.

\begin{tabular}{lcccccc}
\hline & \multicolumn{2}{c}{$f_{\min }$} & $f_{0}$ & \multicolumn{2}{c}{$f_{\max }$} \\
$\phi_{0}$ & $\overline{\mathrm{SF}}$ & $\overline{\Gamma_{y y}}$ & $\bar{\varepsilon}$ & $\overline{\Gamma_{y y}}$ & $\overline{\Gamma_{y y}}$ & $\bar{\varepsilon}$ \\
\hline 0 & 1.17 & 0.917 & 5.2 & 0.951 & 0.948 & 7.9 \\
50 & 1.12 & 0.915 & 5.0 & 0.950 & 0.949 & 8.2 \\
100 & 1.12 & 0.912 & 3.8 & 0.950 & 0.948 & 8.6 \\
150 & 1.10 & 0.908 & 4.3 & 0.948 & 0.949 & 8.8 \\
200 & 1.07 & 0.907 & 3.6 & 0.948 & 0.949 & 8.6 \\
250 & 1.10 & 0.907 & 4.3 & 0.947 & 0.945 & 8.1 \\
300 & 1.16 & 0.913 & 4.7 & 0.948 & 0.947 & 7.5 \\
350 & 1.19 & 0.917 & 5.4 & 0.950 & 0.947 & 8.0 \\
\hline
\end{tabular}

For illustrative purpose, the procedure is detailed only for the $M R C$ strategy. The results obtained with the four cell selection approaches are summarized in the next section.

Table 1 reports the indicators associated to each synthesized $M R C$ layout. Note that $\bar{\varepsilon}\left(f_{0}\right)$ is not reported in Table 1 as it is null.

The relatively low values of $\overline{\Gamma_{y y}}$ shown in Table 1 might be related to cross-polarization generated by cells distant from the center of the array, with high incidence angle $\left(38^{\circ}\right)$. To mitigate this phenomenon, nonsymmetrical cells could be used. $\overline{\Gamma_{y y}}$ is an arbitrary indicator used for layout selection and is not directly related to the losses and cross-polarization levels radiated by the reflectarray (does not account for the tapering on the array). It can be noticed that the $\overline{\Gamma_{y y}}$ parameter is not really affected by the $\phi_{0}$ value. This is consistent with the used $M R C$ strategy that aims at maximizing $\left|\Gamma_{y y}\right|$ for each array element. However, larger variations can be observed for the nonoptimized indicators $(\overline{\mathrm{SF}}$ and $\bar{\varepsilon})$.

As a consequence, the selection consists in choosing the $\phi_{0}$ value that results in the best trade-off among these indicators. For the $M R C$ strategy, the layout obtained with $\phi_{0}=$ $50^{\circ}$ is selected.

4.2.2. Selected Layouts. The same layout selection is applied for each strategy. The RF performance of the four resulting layouts are summarized in Table 2. It can be observed that 


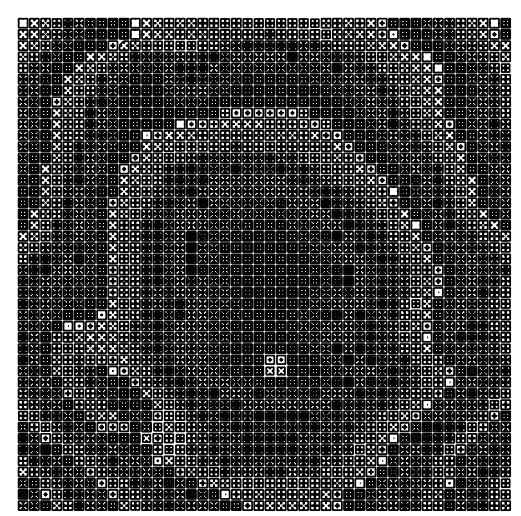

(a) MRC layout

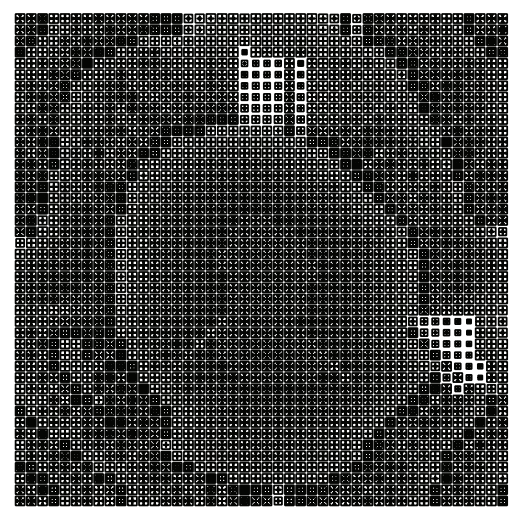

(c) G layout

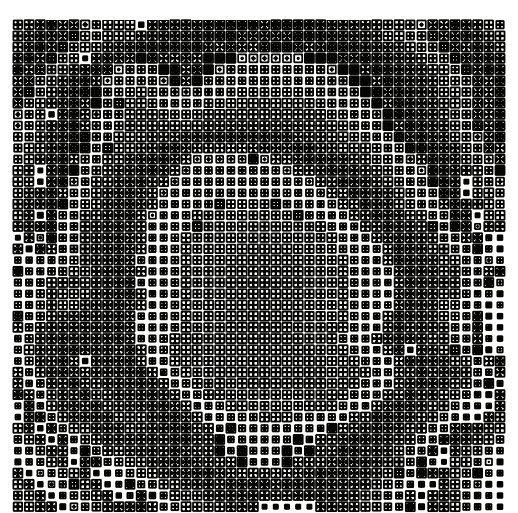

(b) $D$ layout

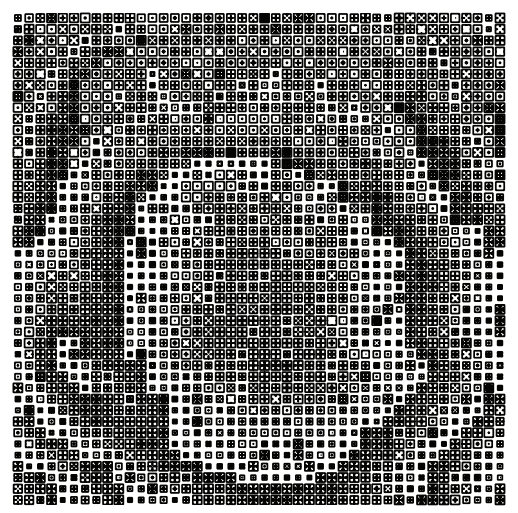

(d) $R$ layout

FIGURE 7: Visualization of the four selected layouts.

TABLE 2: Indicators of the four layouts synthesized with the four cell selection strategies.

\begin{tabular}{lccccccc}
\hline & & & \multicolumn{2}{c}{$f_{\min }$} & $f_{0}$ & \multicolumn{2}{c}{$f_{\max }$} \\
Name & $\phi_{0}$ & $\overline{\mathrm{SF}}$ & $\overline{\Gamma_{y y}}$ & $\bar{\varepsilon}$ & $\overline{\Gamma_{y y}}$ & $\overline{\Gamma_{y y}}$ & $\bar{\varepsilon}$ \\
\hline$M R C$ & 50 & 1.12 & $\mathbf{0 . 9 2}$ & 13.6 & $\mathbf{0 . 9 0}$ & $\mathbf{0 . 9 5}$ & 9.5 \\
$D$ & 100 & 0.97 & 0.86 & $\mathbf{1 0 . 4}$ & 0.89 & 0.89 & 7.5 \\
$G$ & 100 & $\mathbf{0 . 1 7}$ & 0.82 & 38.1 & 0.77 & 0.87 & 29.6 \\
$R$ & 150 & 1.41 & 0.85 & 32.7 & 0.79 & 0.83 & 31.8 \\
\hline
\end{tabular}

each strategy leads to a layout that optimizes its own indicator. This characteristic is highlighted by bold numbers in Table 2. The bad RF performance of the $R$ layout confirms the interest to use a specific cell selection strategy.

The four layouts are depicted in Figure 7(a). As expected, the $G$ layout has the smoothest geometrical variations to provide the best accuracy between simulation and measurement. Nevertheless, the accuracy of the prediction also relies on the number of resonant cells in the array: mutual coupling is larger for cells close to resonance [7] which makes these cells more sensitive to their actual environment. The $R$ layout has large geometrical variations distributed all over the panel while for $M R C$ and $D$ layouts, variations are localized only at transition areas. This regularity results from the restricted cell choice imposed by the $M R C$ and $D$ approaches.
Indeed, the $R$ layout is synthesized with only one criterion whereas the $M R C$ and the $D$ layouts are optimized with two criteria. A zoom of a 12 by 12 array elements area on the layouts is presented in Figure 8 to show more clearly the evolution of the geometry of the chosen cells in the four layouts (Figure 7).

\section{Measurement Results}

The chosen layouts have been manufactured and measured. This section compares the results in order to assess the proposed strategies.

5.1. Radiation Patterns at the Central Frequency. The required radiation pattern is presented in Figure 9. We can observe one main beam for the continental USA, Canada, and Puerto-Rico and another one for Hawaii. Figures 10(a)$10(\mathrm{~d})$ show the superimposition of the required and measured radiation patterns of the four manufactured layouts for a conical view of the earth $\left( \pm 8^{\circ}\right.$ angles $)$.

The measured radiation patterns of the $M R C, D$, and the $G$ layouts match the required coverage quite well regarding the small size of the considered antenna while for the $R$ layout, large differences occur. For this layout which presents the largest geometrical irregularities, the local periodicity assumed in the simulation process is strongly violated. This 


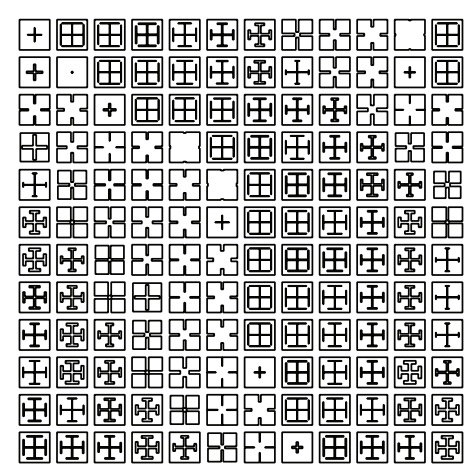

(a) MRC layout

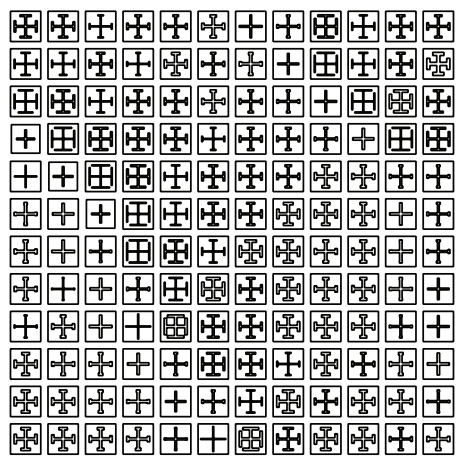

(c) G layout

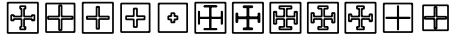

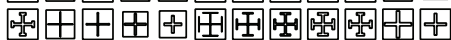

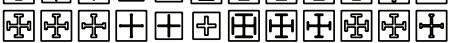

国国国圆四田田田国国国四

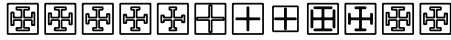

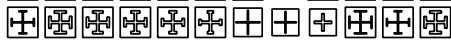

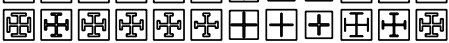

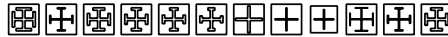

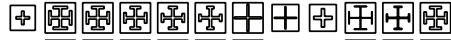

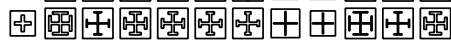

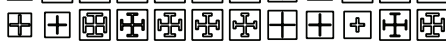

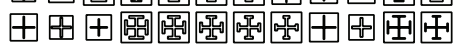

(b) D layout

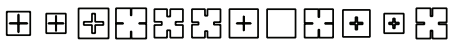

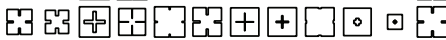

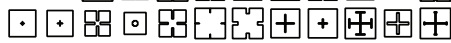

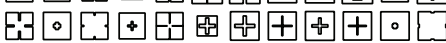

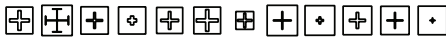

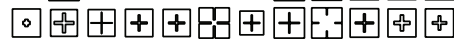

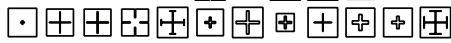

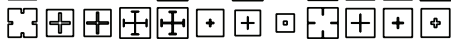

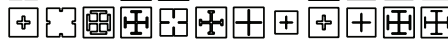

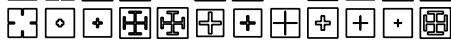

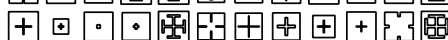

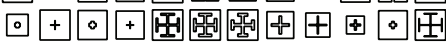

(d) $R$ layout

Figure 8: Zoom in on the top right area of the four layouts (12 by 12 elements area).

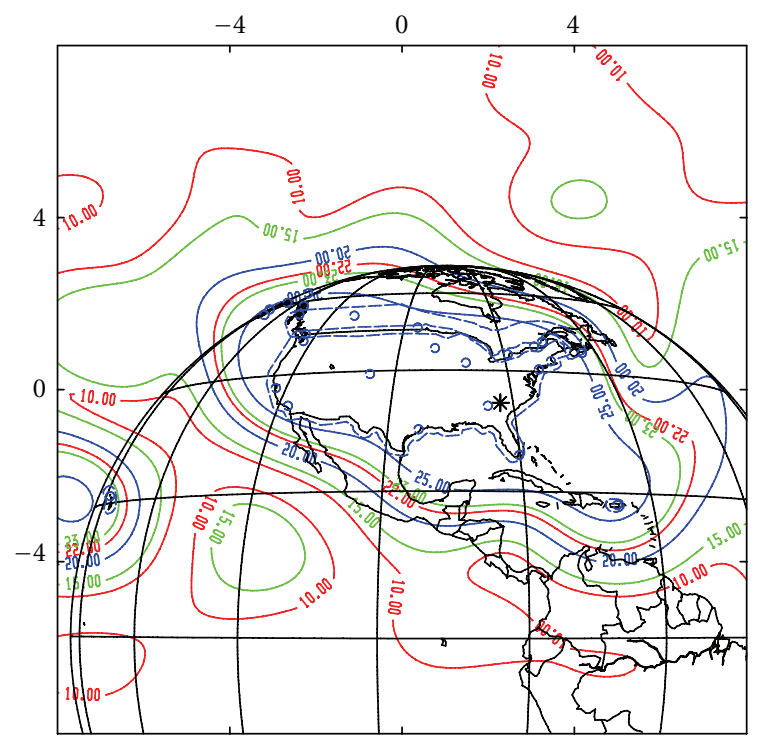

FIGURE 9: Required radiation patterns in the direct polarization at 14.25 GHz.

results in poor RF performance which confirms the necessity to use a specific cell selection process. As a consequence, this layout is no longer studied in the following.

5.2. Losses and Cross-Polarization. Table 3 summarizes the measured ohmic losses for the three layouts at the central
TABLE 3: Measured losses of three manufactured layouts.

\begin{tabular}{|c|c|c|c|}
\hline \multirow[b]{2}{*}{ Name } & \multicolumn{3}{|c|}{ Measured losses $(\mathrm{dB})$} \\
\hline & $14 \mathrm{GHz}$ & $14.25 \mathrm{GHz}$ & $14.5 \mathrm{GHz}$ \\
\hline$M R C$ & 0.5 & 0.2 & 0.1 \\
\hline$D$ & 1.4 & 1.2 & 1.27 \\
\hline$G$ & 0.63 & 0.5 & 0.53 \\
\hline
\end{tabular}

frequency and the extremities of the band. The $M R C$ layout does not only provide a good spatial coverage, it also exhibits the lowest losses. This is consistent with the optimization of $\left|\Gamma_{y y}\left(f_{0}\right)\right|$ performed for this layout. The losses exhibited by the $D$ layout are high compared to the other measured layouts. This phenomenon is explained by the use of a larger number of dispersive cells in order to match the phase law requirements over the frequency band.

Table 4 summarizes the maximum measured crosspolarization level for the three layouts. The cross-polarization levels remain under $-25 \mathrm{~dB}$ for the $G$ and $M R C$ layouts whereas for the $D$ layout, it goes up to $-22.4 \mathrm{~dB}$ at the lower frequency. However, these levels are suitable with the specifications for all cases.

5.3. Radiation Patterns over the Frequency Band. Figures 11(a)-11(f) show the superimposition of the required and measured radiation patterns at 14 and $14.5 \mathrm{GHz}$ for the conical view of the earth. 
DEG (EST)

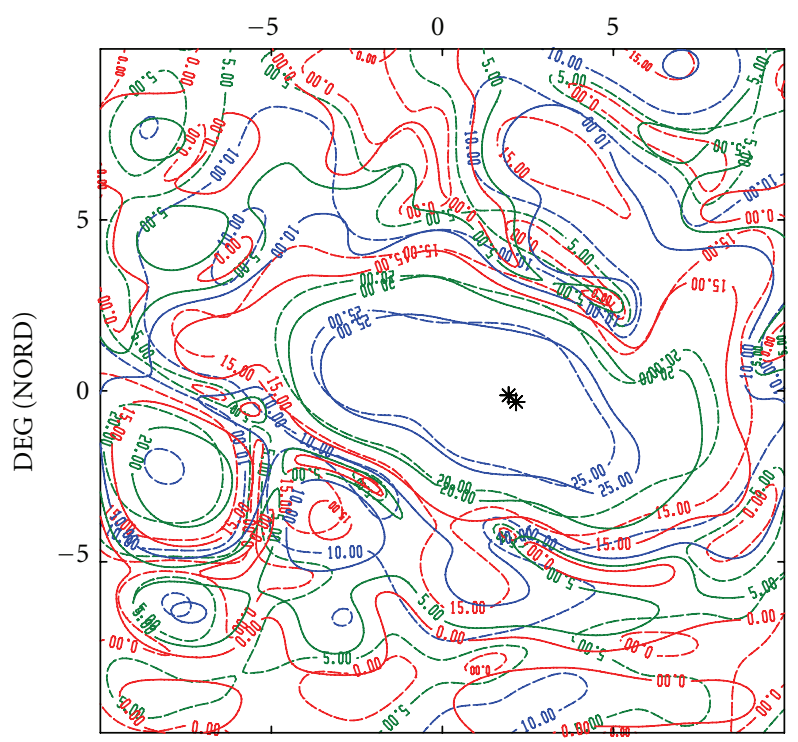

(a) MRC layout

DEG (EST)

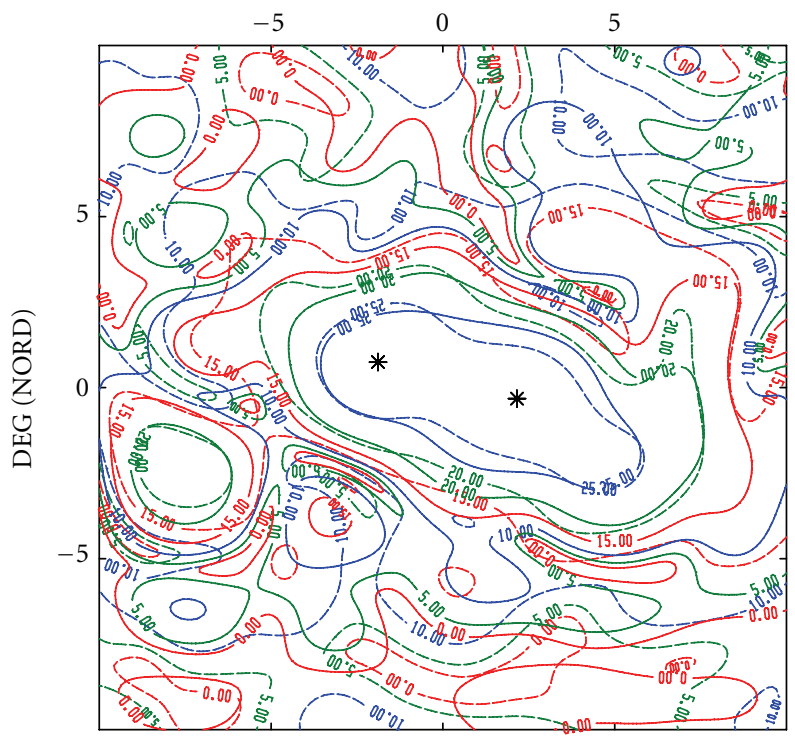

(c) G layout

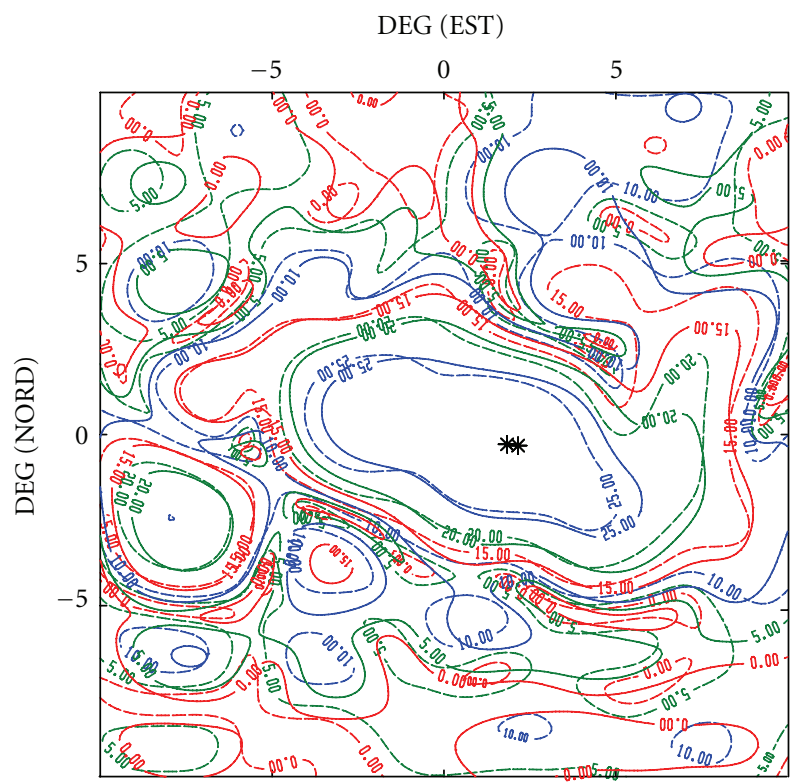

(b) D layout

DEG (EST)

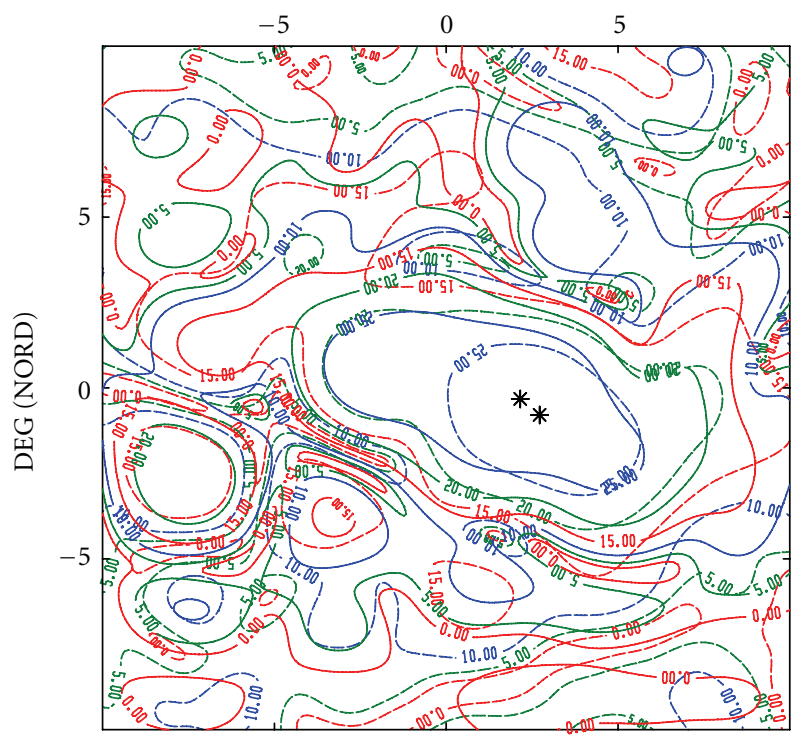

(d) $R$ layout

FIGURE 10: Superimposition of the required (solid) and measured (dashed) radiation patterns at $14.25 \mathrm{GHz}$ in the direct polarization.

TABLE 4: Measured cross-polarization of three manufactured layouts.

\begin{tabular}{lccc}
\hline & \multicolumn{3}{c}{ Measured cross-polarization $(\mathrm{dB})$} \\
Name & $14 \mathrm{GHz}$ & $14.25 \mathrm{GHz}$ & $14.5 \mathrm{GHz}$ \\
\hline$M R C$ & -25.7 & -26.3 & -26.8 \\
$D$ & -22.4 & -25.4 & -27.1 \\
$G$ & -27.3 & -26.9 & -26.6 \\
\hline
\end{tabular}

These layouts exhibit quite stable radiation patterns over the frequency band. As expected, the $D$ layout has a very good matching with the required coverage at the extremities of the frequency band. However, in the present case, the best performance over the frequency band are exhibited by the $M R C$ layout. It can be explained because, unlike the other strategies, it is not a "phase only synthesis" as the module of the direct reflection coefficients are also optimized. It should be noticed that the small size of the breadboard is certainly favorable to the $M R C$ layout. In a larger array, the frequency dispersion would be higher and the improvement brought by the $D$ strategy would be more obvious.

5.4. Radiation Pattern of the MRC Layout. The two main cut views corresponding to the $M R C$ layout are presented in 


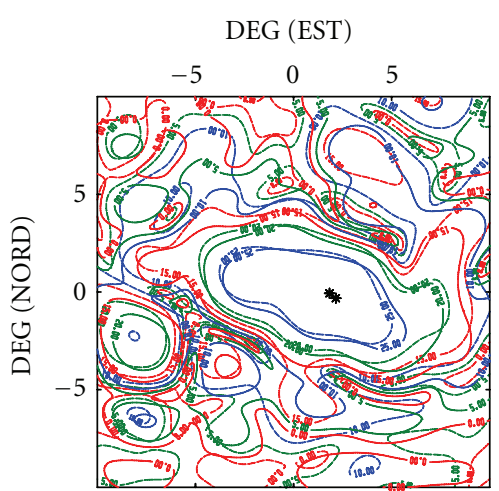

(a) $M R C$ layout at $14 \mathrm{GHz}$

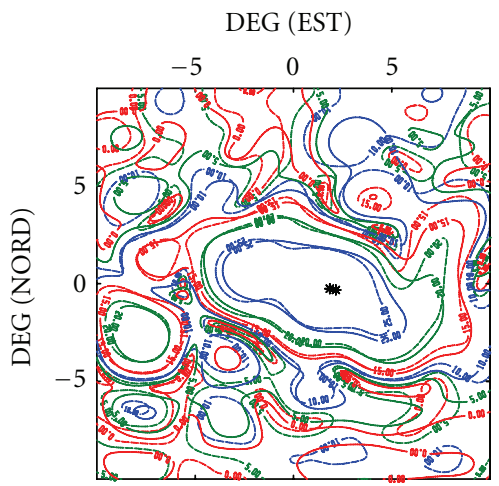

(c) $D$ layout at $14 \mathrm{GHz}$

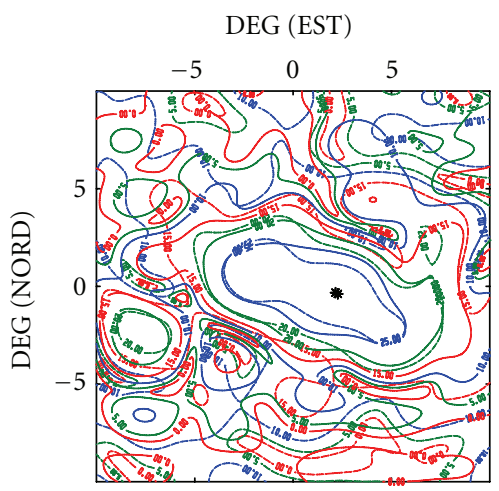

(e) $G$ layout at $14 \mathrm{GHz}$

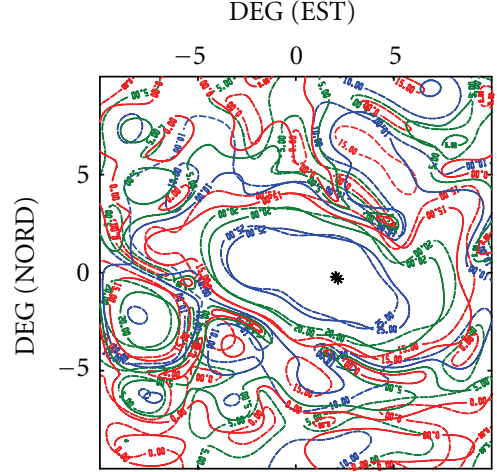

(b) $M R C$ layout at $14.5 \mathrm{GHz}$

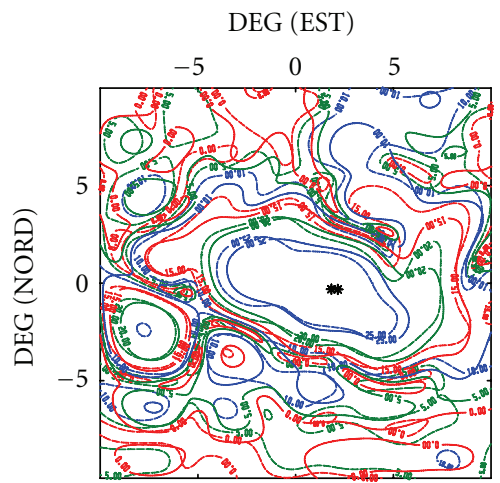

(d) D layout at $14.5 \mathrm{GHz}$

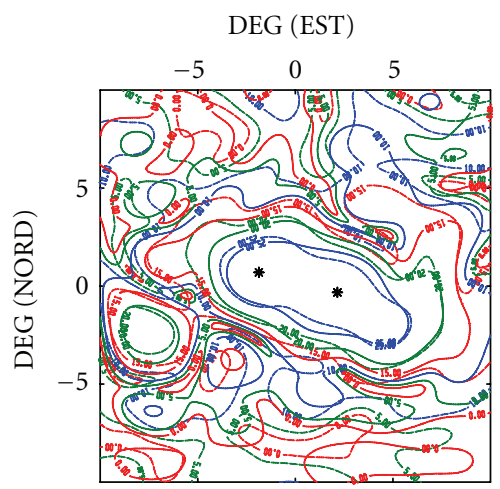

(f) $G$ layout at $14.5 \mathrm{GHz}$

FIGURE 11: Superimposition of the required (solid) and measured (dashed) radiation patterns in the direct polarization of synthesized layouts.

Figures 12 (a) and 12(b) at $14.25 \mathrm{GHz}$ for the direct and the cross-polarizations.

The good coverage matching is confirmed by a maximum gain difference in the direct polarization of $1.5 \mathrm{~dB}$ between the required and the measured ones within the $-10 \mathrm{~dB}$ bandwidth.

\section{Conclusion}

In this paper, optimization strategies have been proposed to select the geometry of the radiating cells when designing a reflectarray. They have been applied to improve the performance of a dual-polarized reflectarray with slot-loaded patches. It has been shown that such advanced synthesis procedures are required to take full benefit of the numerous geometrical degrees of freedom provided by this radiating cell. Comparisons of measured performance have confirmed the superiority of the optimization strategies combining phase and magnitude goals. The corresponding $M R C$ layout reveals low losses and a satisfactory cross-polarization level. Moreover, its radiation pattern is quite stable over the considered frequency band $(0.5 \mathrm{GHz}$ centered on $14.25 \mathrm{GHz})$. 


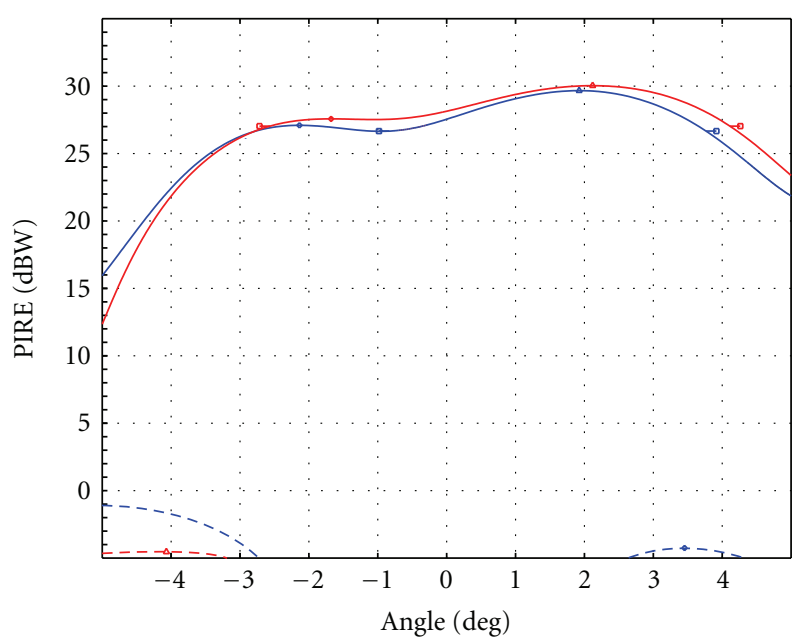

(a) $M R C$ layout at $0^{\circ}$ longitude

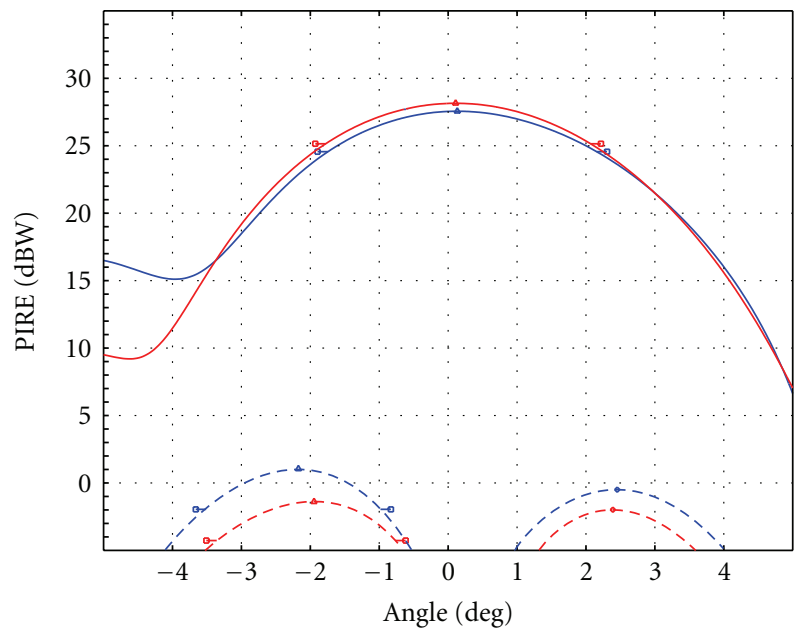

(b) $M R C$ layout at $0^{\circ}$ latitude

FIGURE 12: Superimposition of the required (red) and measured (blue) radiation patterns at $14.25 \mathrm{GHz}$ in direct (solid) and crossed (dashed) polarizations.

\section{Acknowledgment}

The authors would like to thank Dr. R. Chiniard for the support on analysis and measurements.

\section{References}

[1] D. M. Pozar and T. A. Metzler, "Analysis of a reflectarray antenna using microstrip patches of variable size," Electronics Letters, vol. 29, no. 8, pp. 657-658, 1993.

[2] J. Huang, "Bandwidth study of microstrip reflectarray and a novel phased reflectarray concept," in Proceedings of the IEEE Antennas and Propagation Society International Symposium (AP-S '08), vol. 1, pp. 582-585, June 1995.

[3] E. Carrasco, J. A. Encinar, and M. Barba, "Bandwidth improvement in large reflectarrays by using true-time delay," IEEE Transactions on Antennas and Propagation, vol. 56, no. 8, pp. 2496-2503, 2008.

[4] W. Hu, M. Y. Ismail, R. Cahill et al., "Liquid-crystal-based reflectarray antenna with electronically switchable monopulse patterns," Electronics Letters, vol. 43, no. 14, pp. 744-745, 2007.

[5] D. Cadoret, L. Marnat, R. Loison, R. Gillard, H. Legay, and B. Salome, "A dual linear polarized printed reflectarray using slot loaded patch elements," in Proceedings of the 2nd European Conference on Antennas and Propagation (EuCAP'07), pp. 1-5, November 2007.

[6] D. Cadoret, A. Laisne, R. Gillard, L. Le Coq, and H. Legay, "Design and measurement of new reflectarray antenna using microstrip patches loaded with slot," Electronics Letters, vol. 41, no. 11, pp. 623-624, 2005.

[7] M. A. Milon, R. Gillard, D. Cadoret, and H. Legay, "Analysis of mutual coupling for the simulation of reflectarrays radiating cells," in Proceedings of the 1st European Conference on Antennas and Propagation (EuCAP'06), pp. 1-6, November 2006.

[8] J. McSpadden, L. Fan, K. Chang, and J. Huang, "Ka-band beam steering reflectarray study," in Proceedings of the IEEE Antennas and Propagation Society International Symposium, vol. 3, pp. 1662-1665, August 1999.
[9] H. Legay, B. Pinte, M. Charrier, A. Ziaei, E. Girard, and R. Gillard, "A steerable reflectarray antenna with mems controls," in Proceedings of the IEEE International Symposium on Phased Array Systems and Technology, pp. 494-499, October 2003.

[10] D. Bresciani, "Unified approach to the characterization of frequency and polarization selective surfaces," in Proceedings of the IEEE International Symposium Digest of Antennas and Propagation (AP-S '93), vol. 3, pp. 1960-1963, July 1993.

[11] J. A. Encinar and J. A. Zornoza, "Three-layer printed reflectarrays for contoured beam space applications," IEEE Transactions on Antennas and Propagation, vol. 52, no. 5, pp. 11381148, 2004.

[12] L. Marnat, R. Loison, R. Gillard, D. Bresciani, and H. Legay, "Accurate synthesis of a dual linearly polarized reflectarray," in Proceedings of the 3rd European Conference on Antennas and Propagation (EuCAP '09), pp. 2523-2526, March 2009.

[13] L. Marnat, R. Loison, R. Gillard, and D. Bresciani, "Optimization strategies for dual linear polarized reflectarrays," in Proceedings of the 30th ESA Antenna Workshop on Antennas for Earth Observation, Science, Telecommunication and Navigation Space Missions, pp. 1-4, May 2008. 

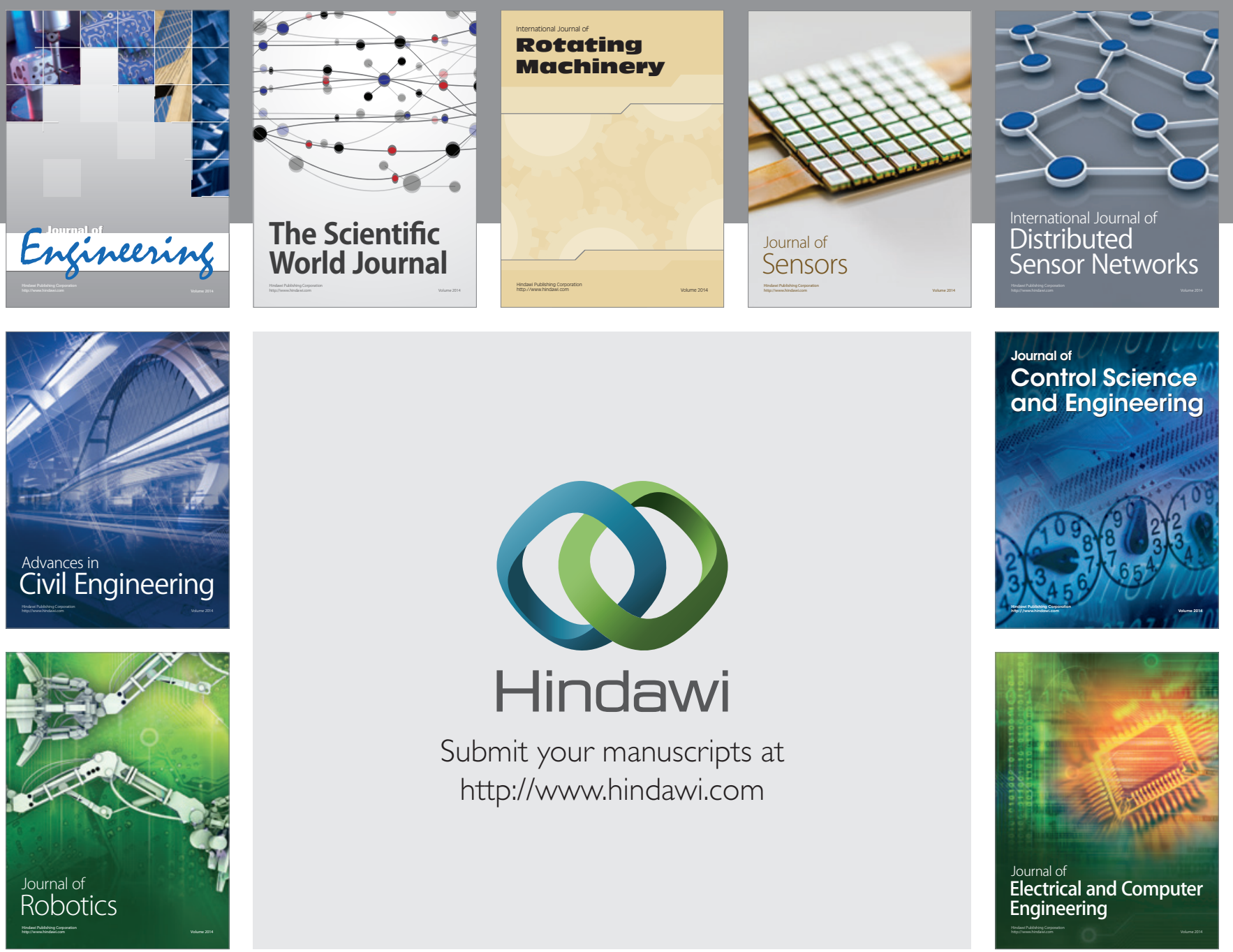

Submit your manuscripts at

http://www.hindawi.com
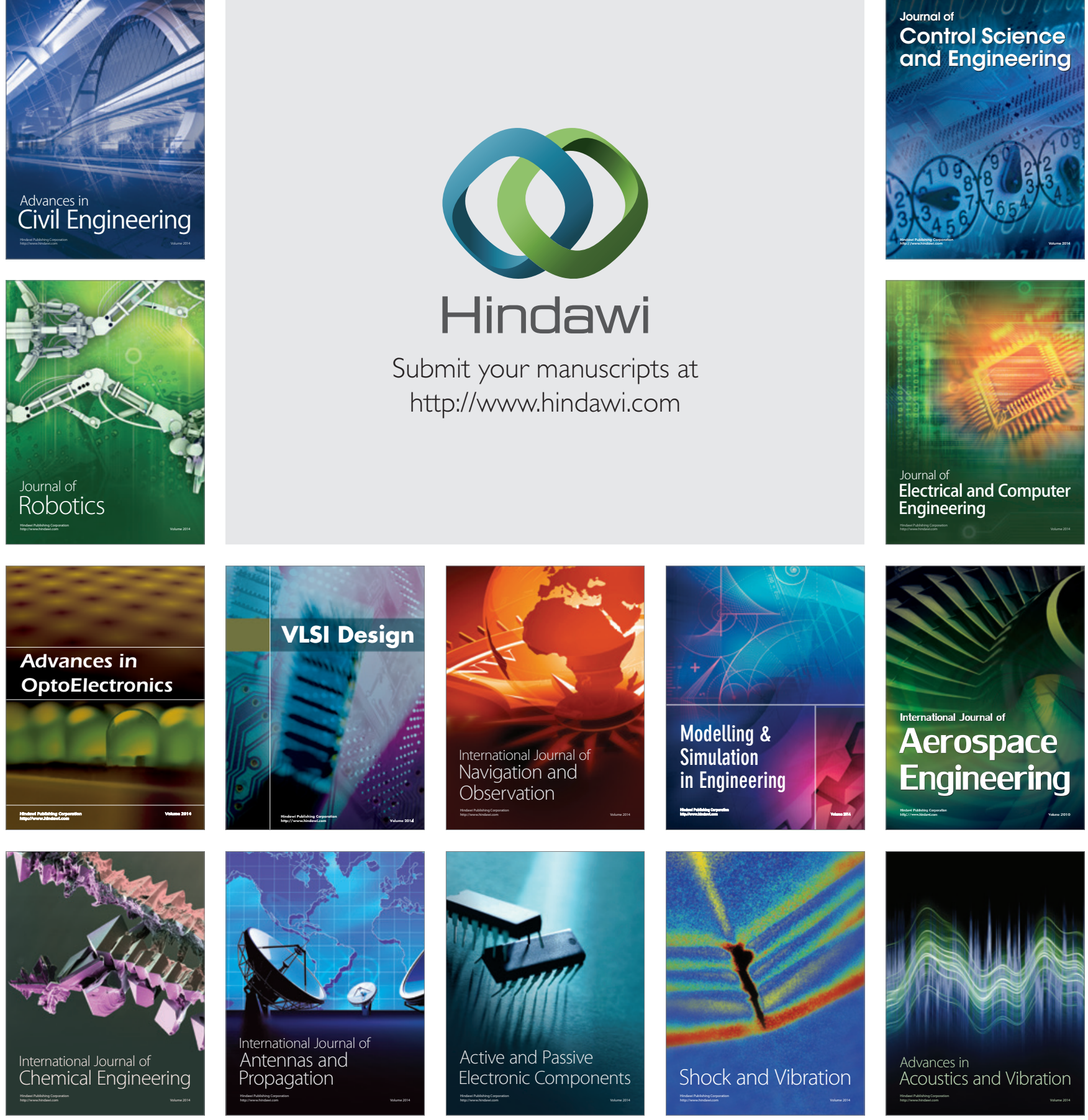